\title{
A clinical practice improvement project on inappropriate intravenous phosphate replacement
}

\author{
Authors: Chaozer Er, ${ }^{\mathrm{A}}$ Joelynn Qiao Bin Tay, ${ }^{\mathrm{A}}$ Hejing Tan, ${ }^{\mathrm{A}}$ Clarissa Hui Wen Aw, ${ }^{\mathrm{A}}$ Nicholas Wong Wai Cheong ${ }^{\mathrm{A}}$ and
} Nurul Atiqa Binte Mohd Tahir ${ }^{\mathrm{A}}$

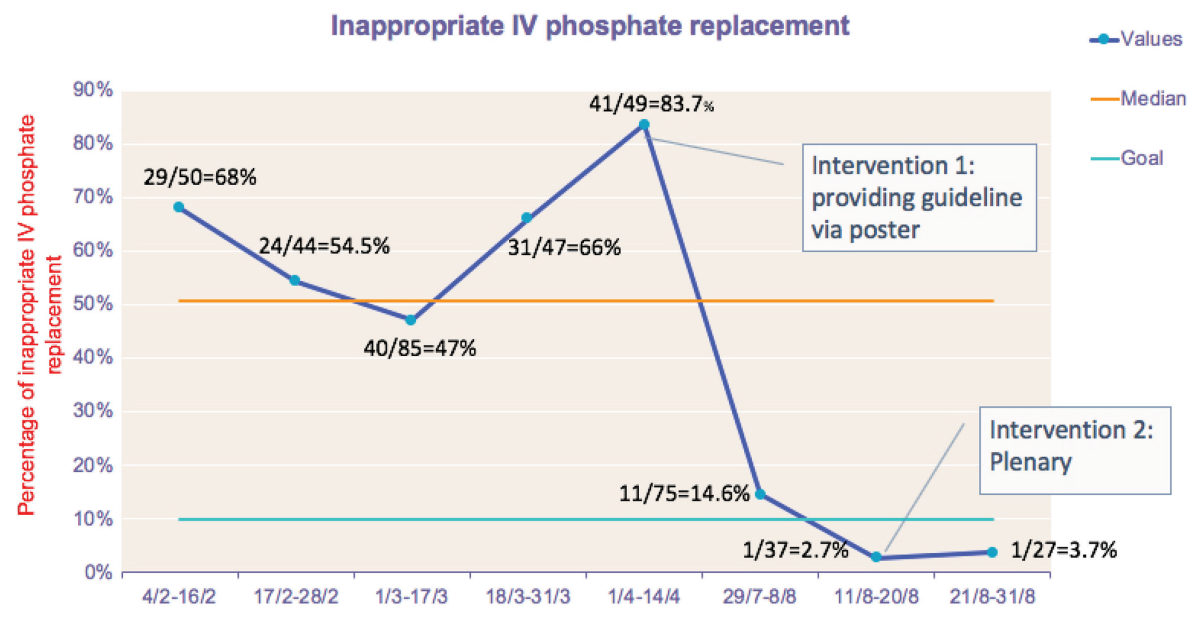

Fig 1. Run chart showing the prevalence of inappropriate intravenous phosphate replacement in two medical wards.

\section{Introduction}

Our hospital's reference range for phosphate is $0.85-1.45$ $\mathrm{mmol} / \mathrm{L}$. Oral replacement is generally adequate for mild and moderate hypophosphataemia $>0.3 \mathrm{mmol} / \mathrm{L}){ }^{1,2}$ Intravenous (IV) phosphate replacement carries many potential side effects and is therefore given for severe hypophosphataemia $(<0.3 \mathrm{mmol} / \mathrm{L})$ only. ${ }^{1,2}$ Inappropriate IV phosphate replacement was common in our ward. We carried out a clinical practice improvement project (CPIP) to address this problem.

\section{Materials and methods}

We retrospectively reviewed the clinical record of all patients with hypophosphataemia admitted to two of our medical wards (total 68 beds) from February to April 2019. 350 hypophosphataemia results were reviewed. They were analysed in blocks of two weeks.

A median of $66 \%$ of hypophosphataemia cases with phosphate $>0.3 \mathrm{mmol} / \mathrm{L}$ were inappropriately given IV phosphate

Author: ${ }^{A}$ Woodlands Health Campus, Singapore
(Fig 1). A previous study reported a prevalence of $85 \%$ in an American hospital. ${ }^{3}$

We formed a team of six members consisting of two pharmacists, three internists and one nurse. A flow chart to describe the process of hypophosphataemia management and a fishbone diagram were constructed.

After two rounds of multi-voting, our Pareto chart showed the top three root causes to address were:

$>$ inadequate published guidance on hypophosphataemia management

$>$ mindset of rapidly correcting laboratory abnormalities

> unfamiliarity with 'oral fleet' phosphate solution.

Two interventions were devised:

> A poster (rolled out on 29 July 2019) providing guidance on hypophosphataemia management and educating doctors and nurses about 'oral fleet' (sodium phosphate solution), as oral phosphate replacement as phosphate tablet is not available in our hospital.

> A plenary session (conducted on 20 August 2019) educating doctors and pharmacists about the rationale and scientific basis of our hypophosphataemia guideline, with the aim of changing their mindset of using IV phosphate for rapid 
correction. Concerns on medication safety, cost and time saving were shared.

We applied plan, do, study, act (PDSA) methodology.

\section{Results and discussion}

With the two interventions implemented, the percentage of inappropriate IV phosphate replacement dropped to $3.7 \%$

(Fig 1). Estimated cost saved per year is about 20,000 GBP. There are other benefits such as nurses' time saved, improved patient comfort and lower risk of medication error.

To ensure sustainability, we propose to

> raise the awareness of hypophosphataemia guideline by introducing it during orientation

$>$ repeat the education talk every 6 months

$>$ set up online module on hypophosphataemia

$>$ upload hypophosphataemia guidelines to the intranet

$>$ encourage nurses to speak up and discuss concerns with doctors about potential inappropriate IV phosphate replacement.

\section{Conclusion}

We have successfully carried out a CPIP to reduce the percentage of inappropriate IV phosphate replacement in our medical wards from $66 \%$ to $3.7 \%$ within 6 months.

\section{Conflicts of interest}

None declared.

\section{References}

1 Royal Cornwall Hospitals NHS Trust. Clinical guideline for the management of hypophosphataemia in adults. NHS, 2017. https://doclibrary-rcht.cornwall.nhs.uk/DocumentsLibrary/ RoyalCornwallHospitalsTrust/Clinical/harmacyClinical GuidelineForTheManagementOfHypophosphataemiaInAdults.pdf

2 Ledere E. Hypophosphatemia treatment and management. Medscape, 2019. https://emedicine.medscape.com/article/242280treatment

3 Hemstreet BA, Stolpman N, Badesch DB, May SK, McCollum M. Potassium and phosphorus repletion in hospitalized patients: implications for clinical practice and the potential use of healthcare information technology to improve prescribing and patient safety. Curr Med Res Opin 2006;22:2449-55. 